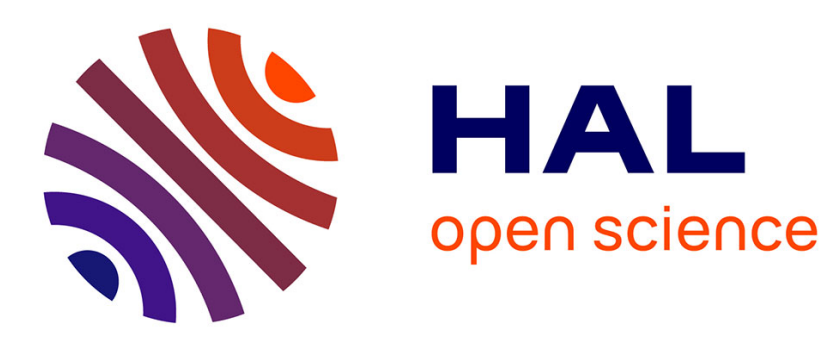

\title{
Two eigenvector determinations in magnesium fluoride
}

R. Almairac

\section{To cite this version:}

R. Almairac. Two eigenvector determinations in magnesium fluoride. Journal de Physique Lettres, 1975, 36 (3), pp.73-77. 10.1051/jphyslet:0197500360307300 . jpa-00231157

\section{HAL Id: jpa-00231157 https://hal.science/jpa-00231157}

Submitted on 1 Jan 1975

HAL is a multi-disciplinary open access archive for the deposit and dissemination of scientific research documents, whether they are published or not. The documents may come from teaching and research institutions in France or abroad, or from public or private research centers.
L'archive ouverte pluridisciplinaire HAL, est destinée au dépôt et à la diffusion de documents scientifiques de niveau recherche, publiés ou non, émanant des établissements d'enseignement et de recherche français ou étrangers, des laboratoires publics ou privés. 


\title{
TWO EIGENVECTOR DETERMINATIONS IN MAGNESIUM FLUORIDE
}

\author{
R. ALMAIRAC \\ Institut Laue-Langevin, B.P. 156, 38042 Grenoble Cedex, France \\ (Reçu le 4 décembre 1974, accepté le 9 janvier 1975)
}

\begin{abstract}
Résumé. - On présente deux mesures de vecteurs de polarisation pour le fluorure de magnésium. Le but de ces mesures est de tester la validité des différents modèles construits pour ce cristal. Bien que les modèles aient été ajustés uniquement sur le spectre de fréquence, les meilleurs modèles du point de vue des vecteurs propres sont ceux qui s'accordent le mieux aux fréquences mesurées.

Abstract. - Two eigenvector measurements are presented for magnesium fluoride. These measurements have been performed in order to test the different models constructed for this crystal. Although the models were fitted to measured frequencies only, it is found that there is good overall agreement between the measured frequencies and the eigenvectors.
\end{abstract}

1. Introduction. - It is a well-established fact in lattice dynamics that knowledge of the phonon frequency spectrum alone is not generally sufficient to obtain the interatomic force constants which generate the vibrations in a crystal. Foreman and Lomer [1] have pointed out that in order to get all these interatomic force constants, it is necessary to measure the eigenvectors $\mathbf{u}\left(\begin{array}{l}\mathbf{q} \\ j\end{array}\right)$ as well as the eigenfrequencies $\omega^{2}\left(\begin{array}{l}\mathbf{q} \\ j\end{array}\right)$ of the dynamical matrix for all modes $(j)$ and for all wavevectors $\mathbf{q}$ in the first Brillouin zone. More precisely, force constants can be determined by Fourier transformation of the dynamical matrix $D(\mathbf{q})$

$$
D(\mathbf{q})=U(\mathbf{q}) \Omega(\mathbf{q}) \tilde{U}(\mathbf{q})
$$

where $U(\mathbf{q})$ is the matrix of the eigenvectors and $\Omega(\mathbf{q})$ the matrix of the eigenvalues $\omega^{2}(\mathbf{q})$. $\tilde{U}$ is the transposed matrix of $U$.

This relation enables us to construct $D(\mathbf{q})$ from experimental determination of $\omega^{2}(\mathbf{q})$ and $\mathbf{u}(\mathbf{q})$. Leigh, Szigeti and Tewary [2] and Cochran [3] have shown that very different sets of force constants can give exactly the same frequencies. From all these sets only one corresponds to the case of the real crystal. In a recent paper Chesser and Axe [4] provide the example of zinc for which two different sets of force constants give normal mode frequencies which agree just as well with the measured dispersion curves but while one set leads to eigenvectors in agreement with experimental data, the other markedly disagrees.

However, for practical reasons it is not possible to carry out systematic measurements of phonon eigenvectors in the whole Brillouin zone. When the crystal contains more than 2 atoms per unit cell, it seems less tedious to use another method. This consists of measuring eigenvectors for some values of $\mathbf{q}$ where they are strongly force-dependent. This information is then included to the data set to which the model is fitted (Brockouse, Becka, Rao and Woods [5]). As an example of the application of this method, we present here two phonon eigenvector measurements for Magnesium Fluoride, a crystal of rutile structure with 6 atoms per unit cell. A rigidion model has been constructed by Katiyar [6] for this crystal and we presented a shell-model together with phonon frequency measurements in a previous paper (Almairac and Benoit [7]).

2. Choice of the Eigenvectors. - For high symmetry crystals such as $\mathrm{CaF}_{2}$, the reduced inelastic structure factor is periodic in $\mathbf{Q}$ space ( $\mathbf{Q}$ : momentum transfer); the corresponding period is called the structure zone (Iizumi [8]). This periodicity property does not hold in the case of $\mathrm{MgF}_{2}$, for the fluorine ion is located in a general position on one of the cell diagonals $\left({ }^{1}\right)$. This lack of periodicity means that intensity measurements for one particular phonon may be made independently in all Brillouin zones of reciprocal space; thus more information may be obtained than is necessary to determine the eigenvector components.

The measured eigenvectors [branch $\Sigma_{1}, \mathbf{q}=(0.4$ 0.40 .), frequency $v=4.4 \mathrm{THz}$ ] and [branch $\Sigma_{3}$, $\mathbf{q}=\left(\begin{array}{lll}0.25 & 0.25 & 0 .\end{array}\right)$, frequency $\left.v=4.2 \mathrm{THz}\right]$ (see [7]), were selected for several reasons;

( $\left.{ }^{1}\right)$ However, for $\mathbf{Q}$ strictly parallel to the crystal axis, the periodicity holds. 
- the instrumental resolution problems are less dramatic for these relatively low frequencies,

- these phonons are well separated from other phonon branches, allowing good background calibration,

- they both have an optic phonon character and each of them is described by 6 parameters (including normalisation).

From symmetry considerations we can obtain the parametric expressions for the eigenvectors (Maradudin and Vosko [9]). The displacement of the atom $\left(\begin{array}{l}l \\ k\end{array}\right)$ for the mode $\left(\begin{array}{l}\mathbf{q} \\ j\end{array}\right)$ is :

$$
\mathbf{u}\left(\begin{array}{l|l}
l & \mathbf{q} \\
k & j
\end{array}\right)=\frac{\mathbf{u}\left(k \mid \begin{array}{l}
\mathbf{q} \\
j
\end{array}\right)}{\sqrt{m_{k}}} \mathrm{e}^{i \mathbf{q x}(l)} \mathrm{e}^{-i \omega t}
$$

$\left(\mathbf{x}\left(\begin{array}{l}l \\ k\end{array}\right)=\mathbf{x}(l)+\mathbf{x}(k)\right.$ position of the $k$ th atom of mass $m_{k}$ in the $l$ th cell).

The dynamical matrix is then defined by :

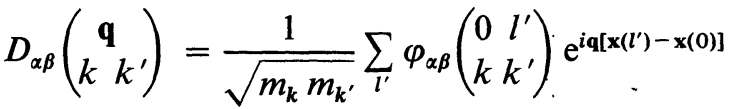

and the $\mathbf{u}\left(\begin{array}{l}\mathbf{q} \\ j\end{array}\right)$ vectors (component : $u_{\alpha}\left(k\left(\begin{array}{l}\mathbf{q} \\ j\end{array}\right)\right)$ are the eigenvectors of the $D$ matrix) :

$$
\left[D(\mathbf{q})-\omega^{2}\left(\begin{array}{l}
\mathbf{q} \\
j
\end{array}\right)\right] \mathbf{u}\left(\begin{array}{l}
\mathbf{q} \\
j
\end{array}\right)=0
$$

Symmetry arguments give :

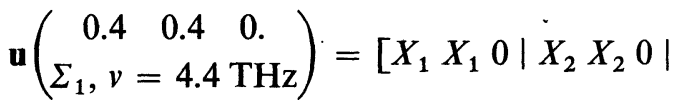

$$
\begin{aligned}
& \left|\left(X_{3}+i X_{4}\right)\left(X_{3}+i X_{4}\right) 0\right| \\
& \left|\rho^{2}\left(X_{3}-i X_{4}\right) \rho^{2}\left(X_{3}-i X_{4}\right) 0\right| \\
& \left|\left(X_{5}+i X_{6}\right) 0\right| \\
& \left.\mid \rho^{2}\left(X_{5}-i X_{6}\right) \rho^{2}\left(X_{5}+i X_{6}\right) 0\right]
\end{aligned}
$$

where $\rho=\mathrm{e}^{i \pi 0.4}$, and for the second phonon :

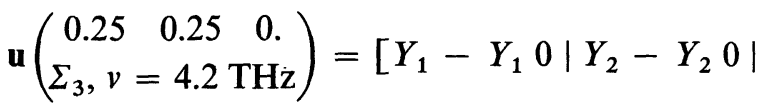

$$
\begin{aligned}
& \left|\left(Y_{3}+i Y_{4}\right)-\left(Y_{3}+i Y_{4}\right) 0\right| \\
& \left|i\left(Y_{3}-i Y_{4}\right)-i\left(Y_{3}-i Y_{4}\right) 0\right| \\
& \left|\left(Y_{5}+i Y_{6}\right)-\left(Y_{5}-i Y_{6}\right) 0\right| \\
& \left.\mid i\left(Y_{5}-i Y_{6}\right)-i\left(Y_{5}+i Y_{6}\right) 0\right] \text {. }
\end{aligned}
$$

The labelling of the six atoms in the unit cell is shown in figure 1. The $X_{i}$ and $Y_{i}$ parameters are all real numbers. For each of these eigenvectors we have chosen the arbitrary phase factor in such a way that the components of the first atom are real. These phonons are not strictly polarised along a fixed direc- tion. However, the first is predominantly longitudinal and the second mainly transversal in character.

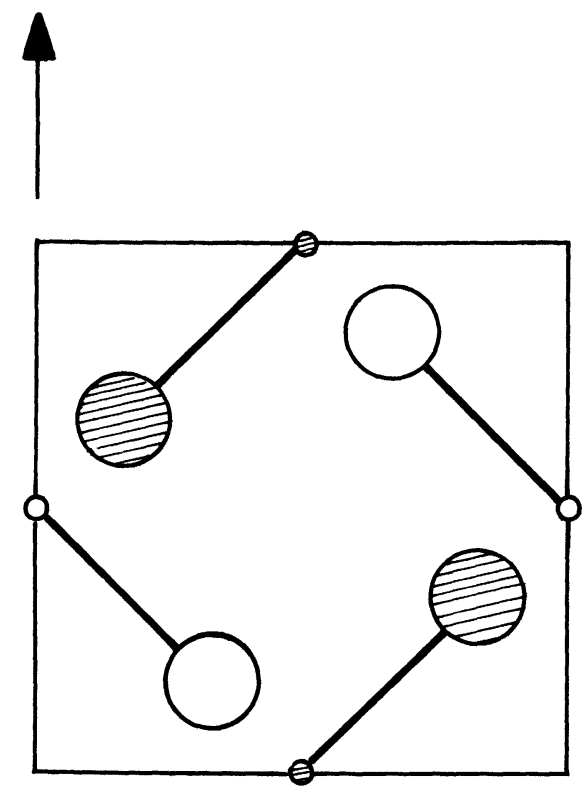

FIG. 1. - Projection of the unit cell of $\mathrm{MgF}_{2}$ perpendicular to the crystal axis. Large circles represent Fluorine atoms and small circles Magnesium atoms. Hatched atoms are at $z=0$, whereas the others are at $z=0.5$.

3. Experimental. - The intensity measurements have been performed on the 3-axis spectrometer IN2 at the HFR of ILL (Grenoble). We used copper (220) as a monochromator and pyrolitic graphite (002) as the analyser. As the momentum transfer $Q$ and the incoming energy were kept constant for all scans, it was necessary to correct them for resolution effects (Dorner [10]). For each eigenvector under investigation, we collected 12 neutron groups. After corrections for resolution, each group was fitted to a Gaussian from which the integrated intensity was analytically deduced. These intensities $J_{j}(j$ from 1 to 12$)$ are directly comparable to the square of the structure factors. For one eigenvector :

$$
\frac{J_{1}}{\left|f\left(\mathbf{Q}_{1}\right)\right|^{2}}=\frac{J_{2}}{\left|f\left(\mathbf{Q}_{2}\right)\right|^{2}}=\cdots=\frac{J_{12}}{\left|f\left(\mathbf{Q}_{12}\right)\right|^{2}}
$$

where $f(\mathbf{Q})$ is the inelastic structure factor :

$$
f(\mathbf{Q})=\sum_{k} \mathrm{e}^{-w_{k}(\mathbf{Q})} \mathrm{e}^{i \widetilde{\mathbf{Q}} \mathbf{x}(k)} \widetilde{\mathbf{Q}} \mathbf{u}\left(k \mid \begin{array}{l}
\mathbf{q} \\
j
\end{array}\right) \frac{b_{k}}{\sqrt{m_{k}}}
$$

$w_{k}(\mathbf{Q})$ Debye Waller factor for atom $k$.

$\mathbf{Q}$ transfer momentum :

$$
\mathbf{Q}=2 \pi \tau-\mathbf{q}
$$

$\tau$, vector of the reciprocal lattice; $b_{k}$, coherent scattering amplitude for atom $k$.

In the expressions (7) and (8) the wavevector $q$ of the eigenvector belongs to the first Brillouin zone. When the eigenvectors $\mathbf{u}(\mathbf{q})$ are calculated from a 
model, the $\mathbf{q}$ range is generally restricted to the irreducible part of the first Brillouin zone. Then the same restriction applies for (7). In order to avoid this restriction, it seems preferable to replace (7) and (8) by :

$\left\{\begin{array}{l}f(\mathbf{Q})=\sum_{k} \mathrm{e}^{-\widetilde{\mathbf{Q} R} M_{k} R \tilde{\mathbf{Q}}} \mathrm{e}^{i \tilde{\mathbf{Q}} \boldsymbol{R} \mathbf{x}(k)} \widetilde{\mathbf{Q}} R \mathbf{u}\left(k \mid \begin{array}{l}\mathbf{q} \\ j\end{array}\right) \frac{b_{k}}{\sqrt{m_{k}}} \\ \text { and } \mathbf{Q}=2 \pi \tau-R \mathbf{q}\end{array}\right.$

$R$ is a $3 \times 3$ matrix associated with one of the operations of the point group of the crystal. In these expressions $\mathbf{q}$ is restricted to the irreducible Brillouin zone, and $R \mathbf{q}$ fills the whole Brillouin zone.

$M_{k}$ is a $3 \times 3$ tensor which takes into account the anisotropy of the Debye Waller factor. For $\mathbf{M g F}_{2}$ these tensors have been deduced from $\mathrm{X}$-ray measurements (Baur [11]).

4. Results. - Using (5) and (6), the parameters $X_{i}$ and $Y_{i}$ are calculated from a fit of $|f(\mathbf{Q})|^{2}((9)$ and (10)) to the measured intensities. Table I gives the values of $X_{i}, Y_{i}$ and of the estimated errors, obtained by this method. We point out the large influence of the Debye Waller factors on the errors $\Delta X_{i}$ and $\Delta Y_{i}$. In other words, precise determinations of $X_{i}$ and $Y_{i}$ need a precise knowledge of the anisotropic Debye Waller factors. Figures $2 b$ and $3 b$ show the comparison

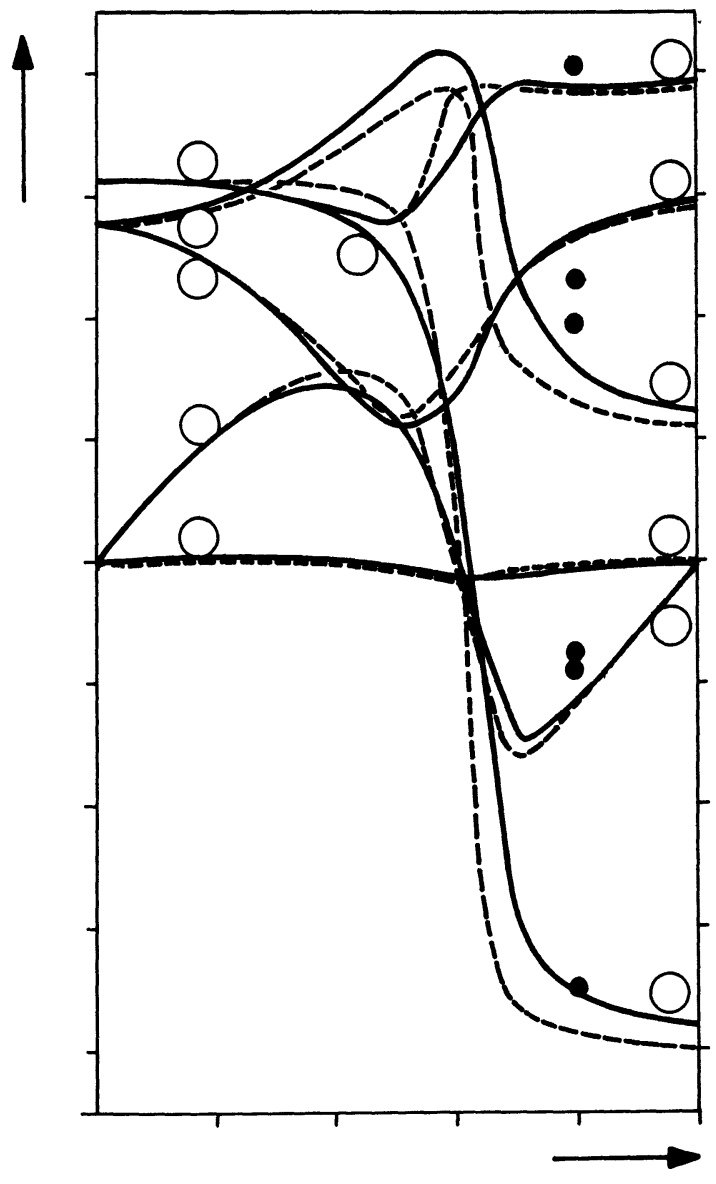

TABLE I

Parameters of the best fit to the intensity measurements 1st phonon $\left(\Sigma_{1}\right)$ 2nd phonon $\left(\Sigma_{3}\right)$

$X_{1}=\overline{0.404} \pm 0.050 \quad Y_{1}=\quad \overline{0.168} \pm 0.040$

$X_{2}=-0.351 \pm 0.030 \quad Y_{2}=0.023 \pm 0.040$

$X_{3}=0.231 \pm 0.030 \quad Y_{3}=0.285 \pm 0.020$

$X_{4}=-0.088 \pm 0.020 \quad Y_{4}=-0.145 \pm 0.030$

$X_{5}=0.20 \pm 0.10 \quad Y_{5}=0.035 \pm 0.020$

$X_{6}=-0.08 \pm 0.18 \quad Y_{6}=-0.363 \pm 0.015$

The normalisation condition $\left(\sum_{k}|\mathbf{u}(k)|^{2}=1\right)$ has been applied for the two phonons.

between the measured intensities (divided by $Q^{2}$ ) $\left(I_{\text {measured }} / Q^{2}\right)$ and the quantities $\left(I_{\text {best fit }} / Q^{2}\right)$ calculated from (9) and (10) in which the $X_{i}$ and $Y_{i}$ are the parameters of the preceding fit. The agreement is good. The « $R$ Factors » :

$$
R=\frac{\Sigma\left|I_{\text {measured }}-I_{\text {best fit }}\right|}{\Sigma I_{\text {measured }}}
$$

are $7.4 \%$ for the first phonon $\left(\Sigma_{1}\right), 8.5 \%$ for the second $\left(\Sigma_{3}\right)$.

FIG. 2. - First phonon $\left(\Sigma_{1}\right)$. a) Variations of the parameters $X_{i}$ of the eigenvector of the $\Sigma_{1}$ mode for $q$ along $(\xi \xi 0)$. The full lines are calculated from model « 5 » and the dashed lines from model « $1 c$ ". The numbers are the indices $i$ of $X_{i}$. Filled circles are experimental points. b) Intensities over $Q^{2}:|F(\mathbf{Q})|^{2} / Q^{2}(\mathbf{q}=0.40 .40) ;---$ measured, calculated with the best fit parameters $X_{i},--$ calculated with $X_{i}$ of model « 5 ». The hatched areas represent the difference between best fit and model « 5 ».

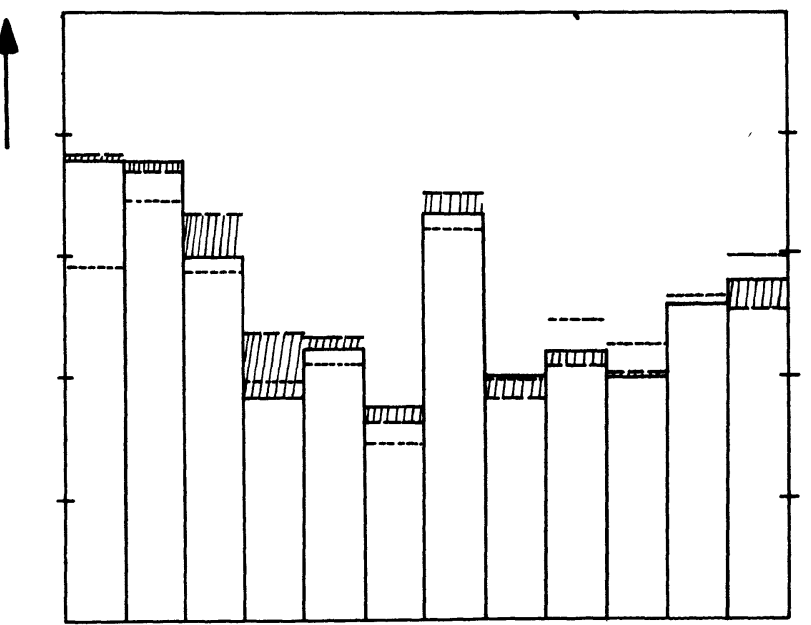



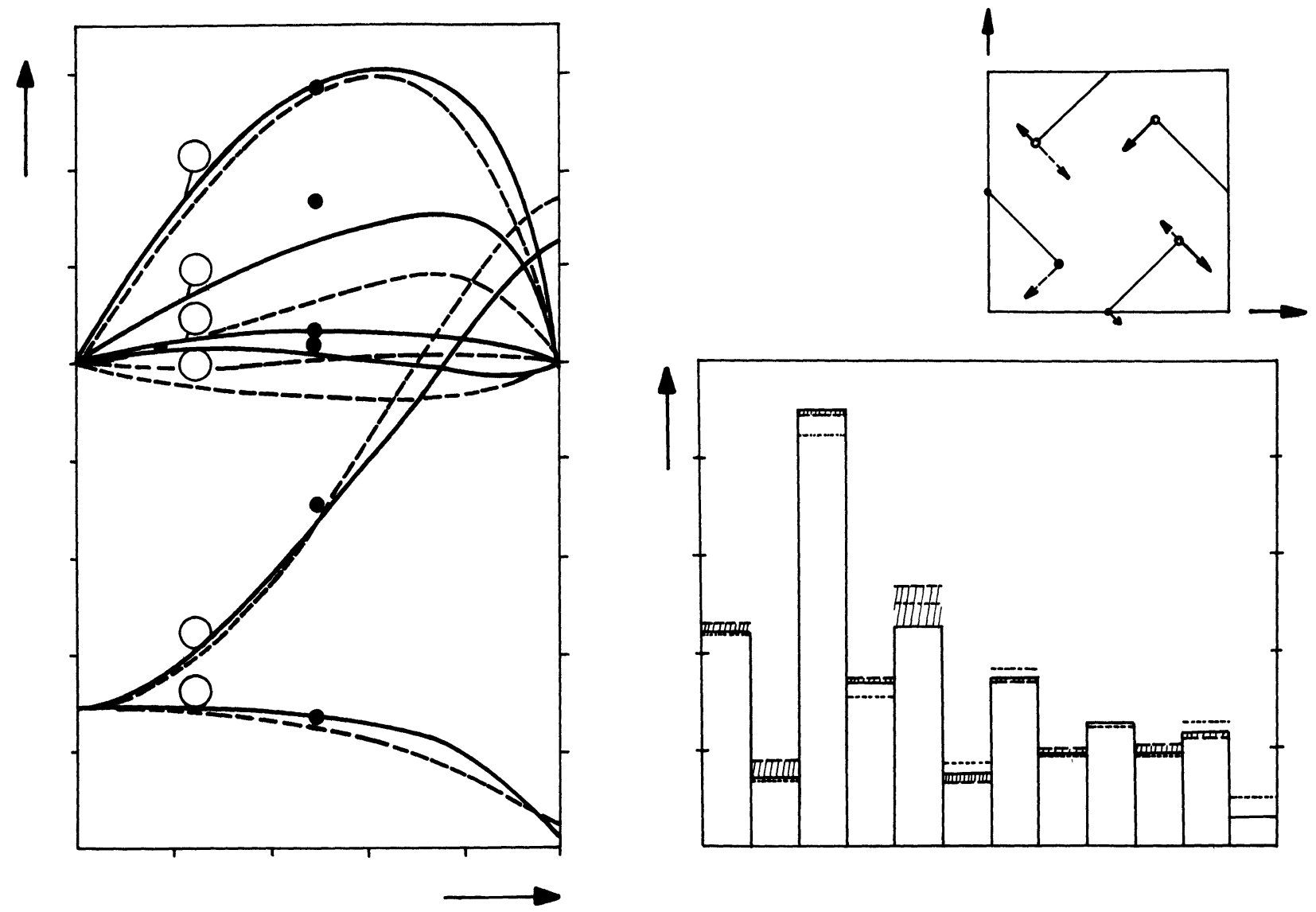

Fig. 3. - Second phonon $\left(\Sigma_{3}\right) . a$ ), b) The same as for figure 2 where $X_{i}$ and $\Sigma_{1}$ are replaced by $Y_{i}$ and $\Sigma_{3}$, respectively. c) Displacements of the atoms for $q=\left(\begin{array}{lll}0.25 & 0.25 & 0\end{array}\right)$. The dashed arrows represent imaginary components.

\section{TABLE II}

Comparison of model predictions with experimental determinations of the eigenvectors

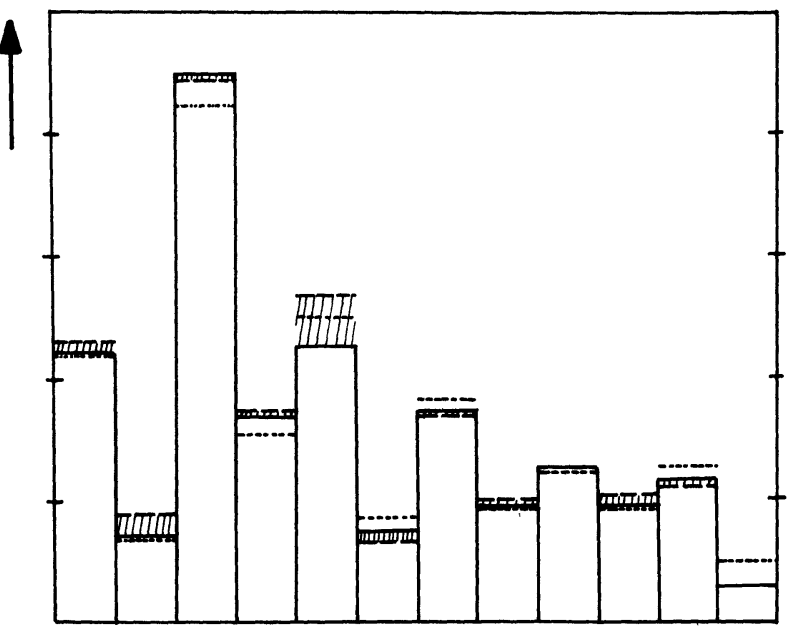

Number of

Models

Rigid ion model « $1 c$ »

Shell model « $2 »(1$ polarizable ion)

Shell model « $3 »(2$ polarizable ions)

Rigid ion model « $1 b »\left(^{*}\right)$

Shell model « 4 » (1 polarizable ion) $(*)$

Shell model « 5 » (2 polarizable ions) $(*)$

\section{Model}

Parameters

-

11

13

6

8

10
$R^{\prime}$ 1st phonon $\left(\Sigma_{1}\right)$

in $\%$

12.0

6.3

6.0

9.2

7.2

6.3

7.4
$R^{\prime}$ 2nd phonon $\left(\Sigma_{3}\right)$

in $\%$

18.5

10.1

10.1

7.6

6.7

5.8

$R$ (experimental determination)

$(*)$ Minimisation of the potential energy per cell is applied for these models ([6] and [7]).

Table II gives the comparison between the experimental determination of the 2 phonon eigenvectors with the predictions of the different models presented in the previous paper [7]. We recall that these models were fitted to phonon frequencies only. The $R^{\prime}$ factor stands for :

$$
R^{\prime}=\frac{\Sigma\left|I_{\text {model }}-I_{\text {best fit }}\right|}{\Sigma I_{\text {best fit }}}
$$

where $I_{\text {model }}$ is calculated from (9) and (10) with the $\mathbf{u}\left(k \mid \begin{array}{l}\mathbf{q} \\ j\end{array}\right)$ given by the models.

We notice that :

- Models « $1 b », ~ « 4$ " and « 5 » for which minimisation of the potential energy has been applied give better results. 
- The agreement is clearly improved on going from rigid ion models to simple shell models_.(one polarizable ion). The improvement still persists from one polarizable ion to two polarizable ion models.

- The model « 5 " is the best compared to the experimental determinations of the eigenvectors : its predictions $\left(R^{\prime}\right)$ are better than the experimental errors $(R)$ for the two phonons.

Figures $2 a$ and $3 a$ compare the variations of the parameters $X_{i}$ and $Y_{i}$ for $\mathbf{q}$ along $(\xi \xi 0)$, as calculated from the best model « 5 » and from the model « $1 c »$. This gives an idea of the dependence of the eigenvectors on the force-constants. However, one must remember that for all these models the interactions are limited to the 4th neighbours (short-range part). Experimental determinations are shown on these figures.

It can be hazardous to draw general conclusions from 2 eigenvector determinations only. At least one can say that for these phonons the models have a real physical sense : the better the agreement with the measured frequencies, the better the agreement with the eigenvectors.

Acknowledgments. - We are indebted to W. G. Stirling, R. Currat and B. Dorner for helpful discussions on this subject.

\section{References}

[1] Foreman, A. J. E. and Lomer, W. M., Proc. Phys. Soc. B 70 (1957) 1143.

[2] Leigh, R. S., Szigeti, B. and Tewary, V. K., Proc. R. Soc. A 320 (1971) 505 .

[3] Cochran, W., Acta Cryst. A 27 (1971) 556.

[4] Chesser, N. J. and AXe, J. D., Phys. Rev. B 9 (1974) 4060.

[5] Brockouse, B. N., BeCKA, L. N., RaO, K. R. and Woods, A. D. B. in Inelastic Scattering of Neutrons in Solids and Liquids (IAEA, Vienna, 1963) Vol. II.
[6] Katiyar, R. S., J. Phys. C 3 (1970) 1693.

[7] Almairac, R. and Benoit, C., J. Phys. C 7 (1974) 2614.

[8] IIzUmi Masashi, J. Phys. Soc. Japan 33 (1972) 647.

[9] Maradudin, A. A. and Vosko, S. H., Rev. Mod. Phys. 40 (1968) 1 .

[10] Dorner, B., Acta Cryst. A 28 (1972) 319.

[11] Baur, W. H. and Khan, A. A., Acta Cryst. B 27 (1971) 2133. 